\title{
Determination the trace elements in Aquilaria sinensis leave By ICP-MS
}

\author{
Yang Yang ${ }^{1,3,4}$, Olagoke Zacchaeus Olatunde ${ }^{1,4}$, Jianping Yong ${ }^{2 *}$, Yuchen Xiao ${ }^{1,3,4}$ and Canzhong Lu ${ }^{1,2 *}$ \\ ${ }^{1}$ Fujian Institute of Research on the Structure of Matter, Haixi Institute, Chinese Academy of Sciences, China \\ ${ }^{2}$ Xiamen Institute of Rare-earth Materials, Haixi Institute, Chinese Academy of Sciences, China \\ ${ }^{3}$ ShanghaiTech University, China \\ ${ }^{4}$ University of Chinese Academy of Sciences, China
}

\begin{abstract}
There are a lot of active components (amino acids, polysaccharides, saponins, alkaloids, flavonoids, organic acids and essential oils) and many trace elements in the leaves of $A$. sinensis. It has been reported that $A$. sinensis leaves were used as functional health tea. But till now, there is no report about the determination of the trance elements in the leaves of $A$. sinensis, we tested the trace elements in the leaves of $A$. sinensis using ICP-MS, to provide the valuable scientific evidence for the development of drinks.
\end{abstract}

Aquilaria sinensis (Lour.) Gilg, a tropical and subtropical evergreen trees, belonging to the family Thymelaeaceae, is endemic and rare medicinal plants in China. A. sinensis can secrete a fragrant defensive black resin when suffering from an injury or infected by fungi. The black resin was named as Chinese eaglewood, which is specific medicine for stomach disease in clinic, and it possess a wide spectrum of biological activities, such as spasmolysis, antihypertensive, antibacterial, sadation, analgesia and so on [1]. The research of $A$. sinensis was mainly focused on the flowers. While the Chinese eaglewood is rare and precious because of its long period of formation. Therefore, injection fungi into the trunk has been carried out to promote the formation of the Chinese eaglewood. In order to comprehensively utilize this resource of $A$. sinensis, much attention has been focused on studying its leaves, especially the leaves after been injected fungi in the trunk of $A$. sinensis, and several healthy drinks have been developed: Wang Keyuan [2] mixed mature and tender leaves of $A$. sinensis with different ratios to prepare different teas for treatment of defecation, detoxification, sleeppromoting and reducing fat; Wang Keyuan [3] also prepared a kind of health product with mature and tender leaves of A. sinensis, Morinda officinalis How, Cistanche deserticola $\mathrm{Ma}$ and Panax quinquefolium in different forms (granule, capsule, tablet, etc.), which can be used for detoxification, nourishing the face, improving sleep, lowering blood sugar and lipid, tonifying kidney, enhancing immunity. Wang Jian et al. [4] prepared a kind of tea with Clerodendranthus spicatus, Aquilaria sinensis leaves and Glycyrrhiza uralensis Fisch, which possess the activities of heat-clearing, detoxicating, promoting liver, drenching, warming kidney. Based on the above results, in this work, we determined nineteen trance elements in three different leaves of A. sinensis (No.1 Natural growth leaves, No. 2 Leaves implanted with fungi, No 3. Non-implanted leaves, all leaves were collected from Taiwan, China) using ICP-MS, to provide scientific evidence for the development of drinks.

The experimental procedure listed below: the three kind different leaves were dried at $60^{\circ} \mathrm{C}$ and powdered respectively. Each sample was weighed the given weight and diluted in $50 \mathrm{~mL}$ volumetric flask using the deionized water after nitrolysis. The trace elements in three different leaves were detected using ICP-MS. The nitrolysis processes see to figure 1 and the results listed in table 1 , which provide the evidence for development of drinks with the leaves of Aquilaria sinensis.

According to scientific research, up to now, there are 18 kinds of essential trace elements related to human health and life. Such as: Fe, $\mathrm{Cu}, \mathrm{Zn}, \mathrm{Co}, \mathrm{Mn}, \mathrm{Cr}$, Se, I, Ni, F, Mo, V, Sn, Si, Sr, B, Rb, As. We can know from table 1 that there are 11 kinds of essential trace elements related to human health and life in he leaves of $A$. sinensis, such as; $\mathrm{V}$, $\mathrm{Cr}, \mathrm{Mn}, \mathrm{Fe}, \mathrm{Co}, \mathrm{Ni}, \mathrm{Cu}, \mathrm{Zn}, \mathrm{As}, \mathrm{Se}, \mathrm{Sr}$. Especially the contents of Mn, $\mathrm{Fe}, \mathrm{Zn}$ and $\mathrm{Sr}$ are rich in the leaves of $A$. sinensis, and $\mathrm{V}, \mathrm{Cr}, \mathrm{Co}, \mathrm{Ni}, \mathrm{As}$, Se are also with higher contents in the leaves of $A$. sinensis. It means that the leaves of $A$. sinensis contains abundant essential trace elements related to human health and life, which can be developed the drinks and health products.

\section{Acknowledgements}

This work was financially supported by the National Natural Science Foundation of China (21875252) and the Project of The Plan of Xiamen Science and Technology (3502ZCQ20171002).

${ }^{\star}$ Correspondence to: VJianping Yong, Xiamen Institute of Rare-earth Materials, Haixi Institute, Chinese Academy of Sciences, China, Tel: +86-591-63173162, E-mail: jpyong@fjirsm.ac.cn

Canzhong Lu, Xiamen Institute of Rare-earth Materials, Haixi Institute, Chinese Academy of Sciences, China, Tel: +86-591-83705794, E-mail: czlu@fjirsm.ac.cn

Key words: Aquilaria sinensis leaves, trace elements, determination

Received: August 12, 2019; Accepted: August 23, 2019; Published: August 26, 2019 


\begin{tabular}{|c|c|c|c|c|c|}
\hline \multirow{2}{*}{ Elements } & \multirow{2}{*}{ Standard curve } & \multirow{2}{*}{$\mathrm{R}^{2}$} & \multicolumn{3}{|c|}{ The content of the elements $(\mathrm{ug} / \mathrm{g})$} \\
\hline & & & No.1 & No. 2 & No.3 \\
\hline $\mathrm{Al}$ & $\mathrm{Y}=0.0128 \mathrm{X}+0.2013$ & 0.9989 & $1.8579 \times 10^{2}$ & $5.9205 \times 10$ & $1.3195 \times 10^{2}$ \\
\hline $\mathrm{Ti}$ & $\mathrm{Y}=0.0203 \mathrm{X}+0.0063$ & 0.9992 & 6.3573 & 4.2760 & 6.3467 \\
\hline $\mathrm{V}$ & $\mathrm{Y}=1.0952 \mathrm{X}+0.0440$ & 0.9992 & 0.4403 & 0.5847 & 0.8295 \\
\hline $\mathrm{Cr}$ & $Y=1.4904 X+0.7925$ & 0.9992 & 2.5941 & 2.2039 & 2.1078 \\
\hline $\mathrm{Mn}$ & $\mathrm{Y}=0.4714 \mathrm{X}+0.2261$ & 0.9994 & $1.0507 \times 10^{2}$ & $4.1486 \times 10^{3}$ & $6.1820 \times 10^{3}$ \\
\hline $\mathrm{Fe}$ & $\mathrm{Y}=1.0349 \mathrm{X}+4.6486 \times 10$ & 0.9988 & $2.0340 \times 10^{2}$ & $3.2262 \times 10^{2}$ & $2.9124 \times 10^{2}$ \\
\hline Co & $\mathrm{Y}=3.0012 \mathrm{X}+0.1006$ & 0.9989 & 0.1013 & 0.4766 & 0.8735 \\
\hline $\mathrm{Ni}$ & $\mathrm{Y}=0.8618 \mathrm{X}+7.3903$ & 0.9988 & 0.5887 & 0.4761 & 1.0901 \\
\hline $\mathrm{Cu}$ & $\mathrm{Y}=2.3178 \mathrm{X}+4.1572 \times 10^{2}$ & 0.9945 & $1.6511 \times 10$ & $<0.000$ & $<0.000$ \\
\hline $\mathrm{Zn}$ & $Y=0.2807 X+1.6809$ & 0.9986 & $1.7542 \times 10^{2}$ & $1.8528 \times 10^{2}$ & $2.2940 \times 10^{2}$ \\
\hline $\mathrm{Ga}$ & $\mathrm{Y}=0.2420 \mathrm{X}+1.0000$ & 0.9995 & 0.0639 & 0.1128 & 0.1598 \\
\hline As & $\mathrm{Y}=0.2098 \mathrm{X}+0.0329$ & 0.9975 & 0.4159 & 1.1240 & 0.4066 \\
\hline $\mathrm{Se}$ & $\mathrm{Y}=0.0045 \mathrm{X}+0.0050$ & 0.9927 & 0.0820 & 0.4115 & 0.4023 \\
\hline $\mathrm{Sr}$ & $\mathrm{Y}=0.0120 \mathrm{X}+0.0023$ & 0.9959 & $4.5023 \times 10^{1}$ & $3.7764 \times 10$ & $4.6574 \times 10$ \\
\hline $\mathrm{Cd}$ & $\mathrm{Y}=0.0111 \mathrm{X}+1.3526 \times 10^{-4}$ & 0.9933 & 0.1952 & 1.5355 & 2.6309 \\
\hline $\mathrm{Sb}$ & $Y=0.0854 X+0.1005$ & 0.9746 & 0.0433 & 0.0504 & 0.0137 \\
\hline $\mathrm{Ba}$ & $\mathrm{Y}=0.0250 \mathrm{X}+0.0264$ & 0.9767 & 6.2973 & $2.1474 \times 10$ & $4.3463 \times 10$ \\
\hline $\mathrm{Tl}$ & $\mathrm{Y}=0.3310 \mathrm{X}+0.0025$ & 0.9878 & 0.0060 & 0.01735 & 0.0143 \\
\hline $\mathrm{Pb}$ & $\mathrm{Y}=0.2715 \mathrm{X}+0.0721$ & 0.9941 & 1.3505 & 1.2660 & 1.2630 \\
\hline
\end{tabular}

Table 1. The content of the trace elements

No.1: Natural growth leaves, No. 2: Leaves implanted with fungi, No. 3: Non-implanted leaves

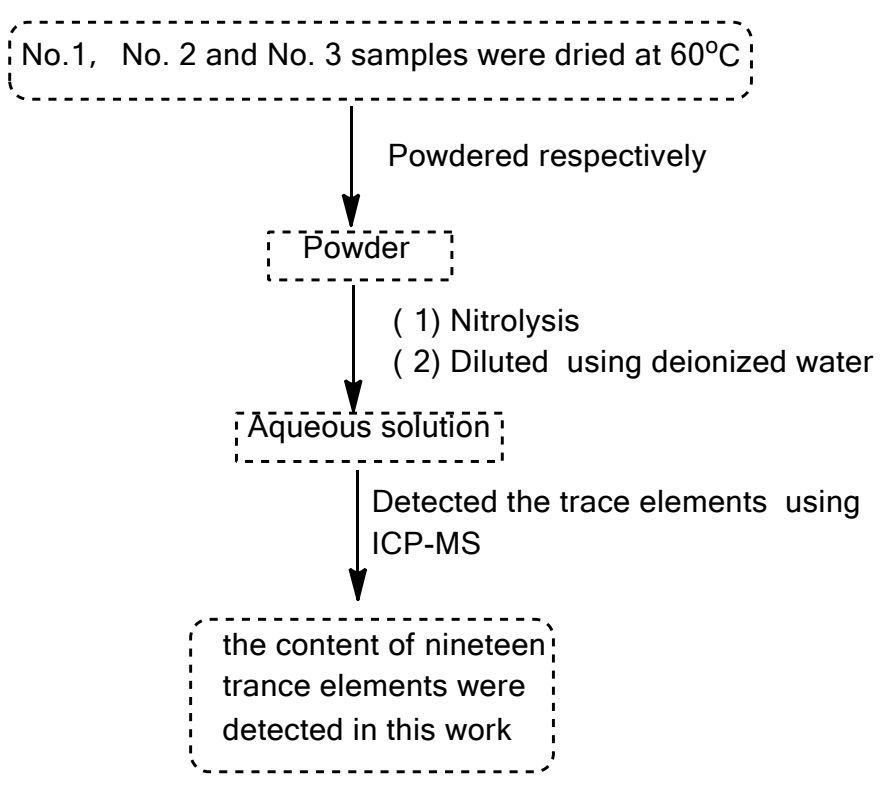

\section{References}

1. Chinese Pharmacopoeia Commission (2015) Pharmacopoeia of the People's Republic of China. Beijing: Science Press, 243.

2. Wang KY (2008) Aloes tea and its Manufacturing process. Chinese patent: 200710029195. 2 .

3. Wang KY (2009) A health food of Aquilaria sinensis leaf and its preparation method. Chinese patent: 200810219857. 7.

4. Wang J, Jin ZY (2007) Aquilaria sinensis and Clerodendranthus spicatus bag tea and its preparation technology. Chinese patent: 200710065657. 6 .

Figure 1. The processes of nitrolysis and detect the trace elements

Copyright: (2019 Yang Y. This is an open-access article distributed under the terms of the Creative Commons Attribution License, which permits unrestricted use, distribution, and reproduction in any medium, provided the original author and source are credited. 